\title{
Electric Field Induced Surface Modification of Au
}

\author{
T. M. Mayer, J. E. Houston, G. F. Franklin, A. A. Erchak, and T. A. Michalske
}

Sandia National Laboratories

Albuquerque, NM 87185-1413

\begin{abstract}
$\underline{\text { Abstract }}$
We discuss the role of localized high electric fields in the modification of Au surfaces with a W probe using the Interfacial Force Microscope. Upon bringing a probe close to a Au surface, we measure both the interfacial force and the field emission current as a function of separation with a constant potential of $100 \mathrm{~V}$ between tip and sample. The current initially increases exponentially as the separation decreases. However, at a distance of less than $\sim 500 \AA$, the current rises sharply as the surface begins to distort and rapidly close the gap. Retraction of the tip before contact is made reveals the formation of a mound on the surface. We propose a simple model, in which the localized high electric field under the tip assists the production of mobile Au adatoms by detachment from surface steps, and a radial field gradient causes a net flux of atoms toward the tip by surface diffusion. These processes give rise to an unstable surface deformation which, if left unchecked, results in a destructive mechanical contact. We discuss our findings with respect to earlier work using voltage pulses in the STM as a means of nanofabrication.
\end{abstract}




\section{Introduction}

There has been considerable interest over the last decade or so in the modification of $\mathrm{Au}$ surfaces by voltage pulse in scanning tunneling microscopy (STM) with special attention being paid to the potential for using this technique for nanostructure fabrication. A series of papers appeared in the early ' 90 s which showed very well controlled formation of nanoscale mounds on Au surfaces by pulsing the tip voltage to several volts at normal STM tip/sample separation distances for periods of hundreds of nsec ${ }^{1-3}$. These authors claimed that the mounds resulted from field evaporation from the tip to the surface. Shortly thereafter, Pascual, et al. ${ }^{4}$ showed that this effect was due to the tip coming into contact with the surface giving rise to very large currents and the mounds resulted from Au migration. The earlier workers maintained their position ${ }^{5}$ claiming that surface deformation would not be fast enough for the mounds to be formed on the times scale of their experiments ${ }^{1-3}$. Additional discussions were not persuasive ${ }^{6}$. Subsequently, several other authors have presented considerable evidence that the formation of mounds and pits results from tip/sample deformation and subsequent mechanical contact ${ }^{4,7-9}$. The evidence for the original suggestion that the process involved field evaporation was the observation that the production of the mounds required a threshold voltage which depended exponentially on the tip-sample separation. But field desorption is a very high-energy process and the fields significantly exceed those required for material deformation. In fact, the threshold fields for bump formation were found to be considerably smaller ${ }^{10}$ than those found for field desorption from field-ion microscopy (FIM) measurements 11. Also, Guo and Thomson found in mound formation using several tip materials (W, Pt-Ir, Mo and Ni) ${ }^{7}$, that the moundformation threshold was independent of the tip materials in spite of the fact that the field evaporation from these materials require FIM field strengths that very widely. However, even after a very systematic study, Koning was led to conclude that none of the existing models for

mound formation were adequate to explain the results ${ }^{10}$. Mendez, et al ${ }^{12}$ have recently shown that electric fields of $0.2 \mathrm{~V} / \AA$ can induce formation of small islands on a $\mathrm{Au}(111)$ surface and 


\section{DISCLAIMER}

This report was prepared as an account of work sponsored by an agency of the United States Government. Neither the United States Government nor any agency thereof, nor any of their employees, make any warranty, express or implied, or assumes any legal liability or responsibility for the accuracy, completeness, or usefulness of any information, apparatus, product, or process disclosed, or represents that its use would not infringe privately owned rights. Reference herein to any specific commercial product, process, or service by trade name, trademark, manufacturer, or otherwise does not necessarily constitute or imply its endorsement, recommendation, or favoring by the United States Government or any agency thereof. The views and opinions of authors expressed herein do not necessarily state or reflect those of the United States Government or any agency thereof. 


\section{DISCLAIMER}

Portions of this document may be illegible in electronic image products. Images are produced from the best available original document. 
propose that field-enhanced diffusion could be the primary mechanism for surface modification in the pulse experiments.

In this paper, we present evidence using the Interfacial Force Microscope (IFM) that significant deformation of the Au surface results from the localized electric field between tip and sample. In order to circumvent the problem presented by operating at tunneling distances in STM, we translate a W tip toward the surface, starting at about $1000 \AA$, under a constant potential and observe the behavior of the current as a function of relative tip/sample separation. Our results clearly show the field emission current varies exponentially as the separation decreases until a certain level of current and field is reached. At this point, the current begins to increase beyond the normal exponential rise, accelerating as the separation continues to decrease. In fact, if the tip approach motion is reversed before contact is made, the Au surface continues to deform toward the tip over a considerable distance. Deformation eventually stops as the tip is withdrawn, leaving a permanent Au mound under the tip.

We use model calculations approximating the tip-sample geometry to obtain the electric fields, the surface stress resulting from the electric field, and to estimate the temperature rise resulting from the field-emission current. We conclude from these results that field desorption, elastic or plastic deformation of the Au surface and surface temperature increases due to the fieldemission current are all unlikely causes for the surface deformation. As in the work of Mendez, et al ${ }^{12}$, we propose a model in which surface diffusion of $\mathrm{Au}$ adatoms in a radial field gradient causes a net flux of atoms toward the tip and growth of a mound, in a manner described by Tsong and Kellogg 11,13,14. In our model a continuous source of adatoms is provided by field-enhanced detachment of atoms from surface steps. This mechanism is similar to that responsible for field induced tip sharpening in field emission and field ion microscopy ${ }^{15}$, and to the recent report by Lyubinetsky, et al ${ }^{16}$ of field induced chemical vapor deposition of $\mathrm{Cu}$ under an STM tip. We perform simulations using this model to estimate the magnitude of the field-induced effects and the initial rates of surface deformation at the conditions of the experiments.

\section{Experimental}


The IFM is a scanning force microscope similar to the atomic force microscope but is distinguished by its use of a stable, self-balancing force sensor ${ }^{17-23}$. Not only does this sensor eliminate the "snap to contact" so prevalent in adhesion, scanning probe and indenter studies but it also represents a zero-compliance sensor, i.e., an applied force does not produce a sensor displacement and no sensor-stored energy results. This is particularly important when studying materials phenomena that involve unstable relaxation processes. In the present work, we use a UHV/IFM, which is capable of sample and probe exchange without breaking vacuum, cleaning by inert ion sputtering (field-emission sputtering for the probe tip) and sample-cleanliness analysis by Auger electron spectroscopy. The sample is a $\mathrm{Au}(111)$ single crystal which is sputter cleaned at room temperature but not annealed. Therefore, some surface roughness can be expected. The probe is formed from a $100 \mu \mathrm{m} \mathrm{W}$ wire by electrochemically etching. The resulting tip radius is nominally $100 \mathrm{~nm}$ as determined by scanning electron microscopy shadow analysis. Since clean W/Au contacts involve massive Au-surface damage 24 , the surface is "located" by an analysis of the behavior of the field-emission current with tip-sample separation. Subsequent to running the experiments of interest, the scale of the current vs. separation is established by forming a mechanical contact.

The experimental results presented here consist of plots of field-emission current, as well as the force exerted on the tip by the electrostatic field, for a tip bias of $-100 \mathrm{~V}$ as a function of relative tip-sample separation. The polarity is such that electrons are emitted from the tip and accelerated to the sample. The software is set to approach the surface at a rate of $25 \AA / \mathrm{sec}$ until a preset field-emission current is reached, then reverse the motion and retract the tip at the same rate. The maximum current value is progressively increased until a time-dependent current behavior is observed. The sample is then translated and the procedure is repeated to obtain a typical mode of behavior. The $1 / \mathrm{d}$ variation of the electrostatic force with relative separation is used to determine a qualitative initial-point separation. After completing the data set, the tip is translated toward the sample from the initial-point current value until contact is established in order to obtain the quantitative separation scale. Subsequent experiments require that the tip be 
recleaned since contact always transfers significant quantities of Au to the W-tip surface ${ }^{24}$. The actual tip-sample separation is also estimated based on calculations of the field emission current and tip radius, compared to the measured emission current, as described in the following section.

\section{Results and Discussion}

The variation of the electrostatic force and field-emission current with respect to tipsample separation for a tip bias of $100 \mathrm{~V}$ is shown in Fig. 1 with a turn around current value of $100 \mathrm{nA}$. The quantitative displacement scale was established as discussed above and we see that the approach turn-around point occurs at about $550 \AA$. The force follows a d $\mathrm{d}^{-1}$ dependence and the current follows an exponential dependence, with no hysteresis between approach and withdrawal. However, if this same type of data is taken at a turn-around current value of 400 nA, from the same starting separation, we obtain the behavior shown in Fig. 2. Up to a tip/sample separation of about $500 \AA$, the force and current behave in the same manner as seen in Fig. 1. However, for smaller separations, the force and current rise more rapidly, accelerating until the tip begins its retraction at about $460 \AA$. At this point, the current remains essentially constant for about the first $50 \AA$ of withdrawal and then gradually settles into an exponential decrease. The approach and withdrawal curves of Fig. $2 \mathrm{~b}$ appear shifted by about $140 \AA$ and the withdrawal data has a slightly higher slope on the semilog plot. The force also follows the expected separation dependence down to values of approximately $500 \AA$ at which point it begins to rise. The force shows some hysteresis at small separation, but returns to the values observed on the approach scan at large separation.

The hysteresis in the emission current (Fig. 2b) shows that the surface has deformed and

a mound with height of about $140 \AA$ has grown beneath the tip. The force measurement indicates that the surface deformation is fairly localized under the tip, however. The force exhibits hysteresis only at the smallest tip-sample gap (Fig. 2a), returning to the originally observed force at larger separation. Since the electrostatic force is the result of a slowly varying, long-range interaction of the tip and sample, the perturbation caused by a localized surface deformation is 
observed only at close separation, while the force observed at large separation is characteristic of the larger-scale tip-sample geometry. From these observations we conclude that the surface deformation consists of a small mound formed directly underneath the tip.

We can get a better picture of the details of this deformation by taking the differences of the approach and withdrawal data of Fig. $2 \mathrm{~b}$ and the expected "no deformation" behavior by extrapolating exponential curves into the non-exponential region, assuming that the behavior in these regions results from the growth of a mound under the tip. These results are show in Fig. 3. Figure $3 \mathrm{a}$ shows surface deformation, determined as outlined above, as a function of time. The time axis is derived by dividing the displacement of Fig. $2 \mathrm{~b}$ by the $25 \AA / \mathrm{sec}$ translation rate. We see here that the surface comes out to meet the tip by about $140 \AA$ over a period of several seconds. The speed of this surface deformation can be found by taking the derivative of the data of Fig. $3 a$ and this result is shown if Fig. 3b. Here we see that the surface velocity rapidly increases to a maximum value of approximately $30 \AA / \mathrm{sec}$ and remains near this value for about one second. In both Figs. $3 \mathrm{a}$ and $3 \mathrm{~b}$, the $(+)$ symbols identify data taken during tip approach while the data taken during tip withdrawal is indicated by $(x)$. Thus, we see that the growth rate of the mound is increasing very rapidly as the current increases. When the current reaches its preset value and tip retraction begins the growth continues to accelerate. However, the growth slows and eventually stops as the tip retracts further. At its maximum, the growth rate of about $30 \AA / \mathrm{sec}$ is larger than the tip retraction rate, so that the mound is continuing to close the gap, even as the tip is retracting.

In contrast to the STM studies, the surface deformation in the present experiments does not involve mechanical tip/sample contact, but is solely due to mass transport of Au under the influence of the only two parameters available, i.e., the electrostatic field and/or the field-emission current. The field gives rise to surface stresses, which can cause both elastic and plastic deformation or field desorption, and its radial gradient can result in forces which encourage surface diffusion toward the axis of the tip. The current, in turn, can facilitate deformation by either surface heating or by electromigration (or both). The key questions are: a) what is the 
major influence in determining the onset of surface deformation, and b) what mechanism of material transport is responsible for growth of the mound.

First we address the possible effects of the emission current. Localized heating of the surface (or even melting) could potentially facilitate transport of material and formation of a cone,

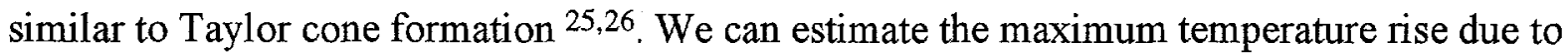
electron impact by considering the energy deposited and transported away from the impact zone by conduction. Solution of the heat transport equation ${ }^{27}$ gives the maximum temperature rise at the center of the beam spot as:

$$
\Delta T \approx \frac{P}{2 \pi^{1 / 2} K \omega}
$$

where $\mathrm{P}$ is the incident power, $\mathrm{K}$ is the thermal conductivity of the sample, and $\omega$ is the beam radius. For field emission from a tip in close proximity to a surface we have shown that the beam radius is approximately half of the tip-sample separation ${ }^{28}$. So for an incident power of $40 \mu \mathrm{W}$ $(400 \mathrm{nA}$ at $100 \mathrm{~V})$, thermal conductivity of $\mathrm{Au}$ of $3.2 \mathrm{Wcm}^{-1} \mathrm{~K}^{12}$, and beam radius of $250 \AA$, we obtain a maximum temperature rise of $<2 \mathrm{~K}$. So beam heating effects appear to be negligible. At these current densities, other possible electron induced transport effects, such as electromigration, can also be discounted. We conclude that current induced processes cannot be responsible for the surface deformation observed.

Electric field effects may include field desorption, elastic or plastic deformation of the solid, or field induced surface transport phenomena. We examine these possibilities below.

As mentioned earlier, the electric field required for field desorption is typically $>1 \mathrm{~V} / \AA$. The maximum field at the sample surface in these experiments (V/d) does not exceed $0.5 \mathrm{~V} / \AA$, so we discount field desorption as a likely mechanism for material transport.

The surface stress resulting from the electrostatic field can be calculated from the expression,

$$
\sigma=\varepsilon_{0}(\mathrm{E})^{2}
$$


where $E$ is the field and $\varepsilon_{0}$ is the permitivity of free space. In order to determine the elastic deformation of the surface under the influence of the field, we must first determine the value of $\mathrm{E}$ as a function of the radial distance from the center of symmetry, i.e., the tip axis. To a good approximation, we find (see below) that the field varies as a Lorentzian function of the form,

$$
E(r)=\frac{V}{d}\left(\frac{1}{\left(\frac{2 r}{w}\right)^{2}+1}\right),
$$

where $r$ is the radius from the center of symmetry, i.e., the tip axis and $w$ is the Lorentzian width parameter. We find $w$ varies with the tip-sample separation $d$ according to the relation,

$$
w=108 d^{1 / 2} .
$$

The maximum surface elastic deformation at the center of symmetry can be found from the expression given by Johnson ${ }^{30}$, p. 53 for a point load,

$$
\delta_{0}=\frac{1-v^{2}}{\pi Y} \int \frac{p(r)}{r} 2 \pi r d r
$$

where $v$ and $Y$ are Poisson's ratio and Young's modulus for $\mathrm{Au}\left(0.44\right.$ and $\left.80 \mathrm{GPa},{ }^{31}\right)$, and $\mathrm{p}(\mathrm{r})$ is the pressure (or stress) distribution given by Eqs. (2) and (3). Substituting and performing the integration results in the expression,

$$
\delta_{0}=\frac{\pi}{4} \cdot \frac{1-v^{2}}{Y} \varepsilon_{0} \cdot\left(\frac{V}{d}\right)^{2} \cdot 108 \cdot d^{1 / 2}
$$


Substituting values appropriate for our situation at the threshold for surface deformation (V=100 volts and $d=400 \AA$ ), results in an elastic deformation value of only about $1 \AA$. Clearly, the surface motion of Fig. $3 \mathrm{a}$ is not effected by elastic deformation under the influence of the field. The next question is whether the electrostatic stress is large enough to cause plastic deformation of the surface? This is easily answered by calculating the maximum stress from Eq. (2) where the maximum field is simply $\mathrm{V} / \mathrm{d}$. The maximum stress comes out to be $43 \mathrm{MPa}$, a value well below the Au yield stress of about $2 \mathrm{GPa}{ }^{31,32}$. So the surface stresses are not large enough to directly cause the surface deformation.

Polarization effects due to the high electric field can alter energies and kinetic barriers for a number of surface processes, including attachment and detachment of atoms at step edges, diffusion of atoms and molecules, dissociation of molecules and small clusters, reconstruction of crystalline surfaces, etc. We suggest that the most likely mechanism for surface modification in this system is field-induced production of mobile surface adatoms by detachment from steps, and preferential diffusion toward the tip in the radial electric field gradient. Tsong and Kellogg 13,14 have shown that polarization of an adatom in an electric field gradient causes a slight bias in the energetic barriers for diffusion, inducing preferential motion toward the direction of higher field. The field induced detachment process has not been investigated in detail, but we will show that polarization effects can alter energetic barriers to detachment sufficiently to provide a source of mobile adatoms.

To explore the feasibility of this proposal, we have constructed a simple kinetic model including polarization effects on the energetic barriers for detachment of Au atoms from surface steps and preferential diffusion in a radial field gradient. We calculate the spatially dependent electric field at the surface, use this field to estimate barriers to detachment and diffusion, and calculate a net flux of material toward the tip. Because we do not know the precise nature of the surface or the precise tip geometry in these experiments, it is difficult to make quantitative comparisons to the experimental data. We use this model only for qualitative exploration of the possible mechanisms. 
To calculate the electric field at the sample, we must know the tip geometry and tipsample separation. In our simulation, we assume the tip is a hemisphere on a conical shank with an angle $20^{\circ}$. We numerically solve Laplace's equation for the potential between the tip and surface, from which we calculate the fields at tip and sample. Knowing the fields, we also calculate the emission current at the tip using the Fowler-Nordheim expression, and the electrostatic force on the tip. Details of the calculation can be found in Mayer ${ }^{28}$.

The effective tip radius and tip-sample separation can be obtained by comparing the field emission current and electrostatic force measured in the experiments to that calculated for various tip radii and separation. The results of this exercise are shown in Fig. 4, where the data of Fig. 1 (no surface modification) are shown along with the calculated emission current and electrostatic force, for a tip with radius of $380 \AA$. The experimental tip-sample separation was matched to that of the calculations to provide the best fit to the emission current. We see from this exercise that the exponential dependence of the emission current and the reciprocal dependence of the force on separation are well characterized by the calculation. We believe that the quantitative disagreement between the calculated and experimental force is due to details of the actual tip geometry. We chose the hemispherical tip radius that best fits the field emission current. However, the current is quite sensitive the presence of small irregularities or asperities on the surface, while the force is determined more by the large-scale structure of the tip and shank. Calculations using more realistic tip geometries would probably give better agreement with both current and force measurements, but this detail is not necessary to explore the main features of our model. For subsequent calculations we assume a tip radius of $380 \AA$, and tip-sample separation estimated from the observed current at which surface deformation begins to occur. For a flat sample surface we find that the radial field at the sample is well characterized by a Lorentzian function, given by equations (3)-(4).

The kinetic model is shown schematically in Fig. 5. Tsong and Kellogg ${ }^{13}$ have shown that high electric fields will alter barriers to surface diffusion. In a field gradient adatoms diffuse toward regions of higher field, with an average drift velocity, $\mathrm{v}_{\mathrm{i}}$. The drift velocity in a constant lateral field gradient is given by: 


$$
v(r)=\frac{4 D_{E=0}}{l}\left(e^{u}-e^{-u}\right),
$$

where

$$
u=\frac{\alpha \beta E l}{k T} .
$$

$\mathrm{D}_{\mathrm{E}=0}$ is the surface diffusion constant in the absence of a field, $\alpha$ is the adatom polarizability, $\beta$ is the radial field gradient, $\mathrm{E}$ is the field and $l$ is the jump length. (Note that this mechanism imposes only a small bias on the normally random diffusion process, yielding a net flux in the direction of higher field.)

Now consider the growth (or etch) rate of an area element, $i$, at radius $\mathrm{r}$ from the center axis (directly under the tip), with inward motion of diffusing adatoms toward the region of higher field. The flux of adatoms diffusing into $i$ across the boundary at $(\mathrm{r}+\Delta \mathrm{r} / 2)$ is given by:

$$
F_{\text {in }}=N_{i+1} v_{i+1} 2 \pi\left(r+\frac{\Delta r}{2}\right),
$$

where $\mathrm{N}_{\mathrm{i}+1}$ is the density of adatoms in the adjacent (outer) area element, $\mathrm{v}_{\mathrm{i}+1}$ is the drift velocity of adatoms for $i+I \rightarrow i$, and $2 \pi(\mathrm{r}+\Delta \mathrm{r} / 2)$ is the outer perimeter of the $i$ th element. The flux of adatoms diffusing out of $i$ across the boundary at $(\mathrm{r}-\Delta \mathrm{r} / 2)$ is given by:

$$
F_{\text {out }}=N_{i} v_{i} 2 \pi\left(r-\frac{\Delta r}{2}\right) .
$$

Then the growth (etch) rate in area element $i$ is:

$$
\frac{d z_{i}}{d t}=\frac{F_{\text {in }}-F_{\text {out }}}{\rho 2 \pi r \Delta r},
$$


where $\rho$ is the atom density of the material.

The mechanism of Fig. 5 also requires source and sink terms for adatoms. The source term, we propose, is the detachment of atoms from surface steps, which will increase the adatom density by:

$$
\frac{d N_{i}}{d t}=k_{d} S_{i},
$$

where $k_{d}$ is the rate constant for atom detachment and $S_{i}$ is the surface step-site density. The rate constant for detachment has the usual Arrhenius form for its thermal dependence, however the activation energy for detachment, $\mathrm{E}_{\mathrm{d}}$, should be field dependent as well. Polarization of an atom at a step in a field will result in a reduction of the activation energy by an amount proportional to the polarizability of the atom and the square of the field, such that:

$$
E_{d}^{\prime}=E_{d}-\frac{1}{2} \alpha E^{2} .
$$

The adatom loss term corresponds to adatoms reattaching to surface steps as they encounter them, with a rate:

$$
-\frac{d N_{i}}{d t}=k_{a} N_{i} S_{i} .
$$

If we assume that every encounter of a diffusing adatom with a step results in reattachment then $\mathrm{k}_{\mathrm{a}}$ is just the diffusion constant for the adatom, $\mathrm{D}$.

To calculate the density of adatoms, $\mathrm{N}_{\mathrm{i}}$, we employ the steady state approximation, which, in the limit of small adatom densities, assumes that the sum of the diffusion fluxes and the attachment and detachment fluxes is zero. Using this approximation and equations (9),(10),(12), and (14) for the fluxes, solving for $\mathrm{N}_{\mathrm{i}}$ gives the adatom density as: 


$$
N_{i}=\frac{N_{i+1} v_{i+1}\left(r+\frac{\Delta r}{2}\right)+k_{d} S_{i} r \Delta r}{v_{i}\left(r-\frac{\Delta r}{2}\right)+k_{a} S_{i} r \Delta r} .
$$

We then use this expression in to calculate the fluxes in Eq. (11) to obtain the growth rate of area element $i$. In the actual calculation we compute the density, $\mathrm{N}_{\mathrm{i}}$, for large $i$, first, where field gradients are negligible, then incrementally to smaller values of $i$.

Inputs to the model include values for the Au surface diffusion coefficient, $\mathrm{Au}$ atom polarizability, activation energy for step detachment, and the surface step-site density. The fieldfree diffusion coefficient for $\mathrm{Au}$ at room temperature is taken to be $3.6 \times 10^{-5} \mathrm{~cm}^{2} \mathrm{~s}^{-1}$ (activation energy of $0.1 \mathrm{eV})^{33}$ and the Au adatom polarizability is $2.5 \times 10^{-24} \mathrm{~cm}^{3} 13,14$. We do not know the activation energy for detachment of a Au atom from a surface step, but assume a value between 0.5 and $1 \mathrm{eV}$. The surface step-site density is a function of the surface slope relative to the (111) plane, as well as the roughness. The experiments were carried out on sputtered, but unannealed samples, whose surfaces were undoubtedly quite rough. As the mound forms the local slope of the surface changes as well. So the surface step-site density is a major unknown in this model. For the sake of calculation we assume a background step-site density on the flat sample of $10^{12} \mathrm{~cm}^{-2}$. As the mound grows the step-site density of straight steps will grow as $\tan \theta /$ ha, where $\theta$ is the local slope, $h$ is the step height, and $a$ is the lattice parameter.

Two factors limit the utility of this model. First, the step and terrace description of the $\mathrm{Au}$ (111) surface limits this treatment to relatively small slope angles. For more highly sloped surfaces, the hopping diffusion process envisioned here may not apply, and the diffusion constant on higher index planes is likely to be lower ${ }^{33}$. Second, the field gradient-induced bias in the surface diffusion results in a net flux of atoms toward higher field, however this is just a small perturbation on the normal random diffusion process. For simplicity, density gradient-driven diffusion fluxes are not included in this model. Since the model predicts an increasing adatom density toward the center, there should be an offsetting density gradient-driven flux away from 
the tip. Both of these limitations make the model strictly valid only for relatively flat surfaces, and small field gradients. This is approximately true at the beginning of the mound formation process, but not after the mound begins to develop. So the following calculations of mound growth rate are limited to the initial stages of growth on a flat surface.

Fig. 6 shows the calculated electric field and the initial growth rate using this model and the parameters defined above, with a step detachment activation energy of $0.6 \mathrm{eV}$, and for a tip with a radius of $380 \AA$, and tip-sample gap of $250 \AA$. Fig. 6 demonstrates that a mound will grow under the tip. At larger distances from the center, a net loss of material is evident. Adatoms produced in this region diffuse toward the tip, but fewer adatoms are diffusing into this region because of smaller fields and lower field gradients at larger distance. The absolute values of growth rate are quite sensitive to values of step-site density, Au polarizability and the detachment activation energy, all of which are not well known. But we are gratified to see that the main features of field-induced surface deformation are well described by this simple model, using reasonable parameter values.

Although we cannot reliably extend this calculation to later stages of mound growth, we can easily show what will happen in a general sense. As the mound forms, the electric field will increase at the top of the mound because of the decreased tip-mound gap, and the curvature of the mound. The radial field gradient will also increase near the top of the mound as the field is concentrated more toward the center of the structure. In this case the growth rate will increase dramatically, as well, leading to an unstable situation, where increasing field yields increasing growth rate, which increases the field ... To show the dramatic nature of this effect, we have calculated the maximum field at the top of a small mound under the tip, and the maximum growth rate for a flat surface as a function of field. These are shown in Figs. 7 and 8. It is quite apparent that a modest increase in the field leads to orders of magnitude increase in growth rate under the tip. If the tip is very close to the sample, as in the STM pulsing experiments, growth of a small mound, even of only a few atomic layers, represents a large relative decrease in the gap, and a correspondingly large increase in the field. So the growth rate can accelerate very rapidly in this arrangement, leading to catastrophic tip-sample contact. 
We should note that in the experiments the mound is observed to grow faster than the tip retraction rate for a time after the tip motion is reversed, shown in Fig. 3b. But the growth rate declines and the mound eventually stops growing, even though the tip-mound gap remains small. The above model suggests that the unstable growth conditions should have caused a tip-sample contact. We suspect that two factors eventually cause growth to slow down and stop in this situation. First, as the tip begins to retract, the electric field at the top of the mound remains high, however the field at large radius decreases. Since the source of diffusing material is primarily the area at large $r$, the supply of adatoms is turned off as the field in this region decreases. Second, as the mound grows, the field becomes more concentrated at the top, with large field gradients extending only to small values of $r$, so there is less of a field induced bias to diffusion at large $r$, the location of most of the mobile adatoms. So in this experimental arrangement, as the mound becomes higher and sharper, the flux of material to continue growing the mound is effectively shut off.

While this simple model qualitatively reproduces the main features of the experiments, we should examine its assumptions to see if it is realistic. The field-enhanced diffusion process has been studied extensively $12-14,16,34-37$ and is generally well accepted. Most notably, Mendez, et al ${ }^{12}$, have observed enhanced surface transport and formation of small islands on the $\mathrm{Au}$ (111) surface under an STM tip, with a static electric field of $0.2 \mathrm{~V} / \AA$.

The field enhanced detachment of atoms from surface steps is less well understood. We know that a source of mobile adatoms is necessary to sustain mound growth, and that polarization effects, which have been shown to alter kinetic barriers to diffusion, should be evident in atom detachment processes as well. Cabibil, et al ${ }^{38}$ have observed dissociation of small islands and an evolution of the step and terrace structure on a Au (111) surface as a result of application of a high electric field, which clearly involves detachment of atoms from steps and migration of adatoms in the presence of a field. We note that the experiments of Cabibil were carried out using a relatively blunt tip, at small tip-sample distances. In this case, electric field gradients under the tip are small, while the magnitude of the field is high. Barriers to detachment and diffusion will be reduced by polarization effects, but the directional diffusion bias induced by 
field gradients should be small. Cabibil observed marked restructuring of the surface step and terrace structure, but no preferential transport of material to the tip central axis. Recent measurements by Kellogg 39 in a field ion microscope also demonstrate enhanced detachment of atoms from edges of small islands on a Pt(111) surface in the presence of electric fields insufficient for field desorption.

The magnitude of the polarization effect on atom detachment in our model seems reasonable. At the highest field in the simulations shown in Fig. 6 the reduction in detachment activation energy due to the polarization term is quite small, approx. $0.02 \mathrm{eV}$. However this is sufficient to supply an adatom flux for mound growth to begin. This degree of change in a surface atom binding energy is consistent with the observations by Swartzentruber 40 of rotation of $\mathrm{Si}$ dimers under an STM tip. Comparable electric fields reduce the activation energy for dimer rotation by $0.02-0.04 \mathrm{eV}$. The FIM measurements of Kellogg 39 also suggest that a field of 0.5 V/Å reduces the detachment barrier for $\mathrm{Pt}$ atoms at a step by approx. $0.04 \mathrm{eV}$. We should also note that our simulations do not account for field enhancement at surface features with large curvature, such as step edges. This microscopic structure of the surface will tend to increase the local field, and enhance polarization effects.

The field effect could include an interaction with surface dipoles, in which case the effect would be polarity dependent ${ }^{11}$. We have not made measurements at opposite polarity (tip positive) because the field emission from the tip is used as a control signal in the experiments. On a metal such as $\mathrm{Au}$, however we expect the dipole contribution to be small ${ }^{14}$, and indeed, Mendez, et al, ${ }^{12}$ did not observe differences due to polarity in their measurements on $\mathrm{Au}$ (111) surfaces.

This mechanism proposed here is consistent with the STM voltage pulse experiments, in which a conducting neck is formed during very short periods of high field. In the experiments of Mamin, et al ${ }^{1-3}$ a neck is formed across a gap of $5-10 \AA$ in a few hundred nanoseconds, at a threshold field on the order of $0.4 \mathrm{~V} / \AA$. The model calculations in Fig. 8 suggest that if the tip is not retracted the unstable growth behavior could conceivably close this small gap in such a short period. More significantly, the model provides insight into the nature of the surface deformation 
process, which can be used to control nanostructure formation. Instead of an uncontrolled, unstable process in which catastrophic tip-sample contact occurs, we have shown that it is possible to make nanostructures of controlled dimension, in a non-contact experiment. The model also suggests a variety of effects based on the relative magnitudes of the field and field gradients, which are influenced by the experimental parameters of tip radius, tip-sample separation and applied voltage. Polarization effects due to the magnitude of the field result in kinetic processes with a hierarchy of activation energies, from enhanced diffusion to field desorption. Directional forces due to field gradients cause preferential transport. Manipulation of the relative magnitudes of the field and field gradients by experimental arrangement gives many opportunities for control of surface restructuring in localized high electric fields.

\section{Acknowledgements}

We wish to thank G. L. Kellogg, H. Cabibil and J. T. Yates for fruitful discussions, and for sharing results prior to publication. Sandia is a multiprogram laboratory operated by Sandia Corporation, a Lockheed-Martin Company, for the U. S. Department of Energy under Contract DE-AC04-94AL85000. 


\section{Figure Captions}

Figure 1. Electrostatic force and field emission current observed simultaneously during tip approach and withdrawal. Tip bias is $-100 \mathrm{~V}$, approach and withdrawal speed is $25 \AA / \mathrm{sec}$, and maximum current set point is $100 \mathrm{nA}$. Force (a) and current (b) show no hysteresis, indicating no surface deformation.

Figure 2. Electrostatic force and field emission current observed simultaneously during tip approach and withdrawal. Tip bias is $-100 \mathrm{~V}$, approach and withdrawal speed is $25 \AA / \mathrm{sec}$, and maximum current set point is $400 \mathrm{nA}$. Both force (a) and current (b) show deviations from expected behavior, and hysteresis upon withdrawal, indicative of surface deformation. Hysteresis in the current indicates a mound approximately $140 \AA$ high has grown under the tip.

Figure 3. Height (a) and growth rate (b) of a mound as a function of time, from the data of Fig. 1b. (+) indicate data obtained during tip approach, and (x) indicate data obtained during tip withdrawal.

Figure 4. Calculated emission current and electrostatic force (o), compared to experimental measurements $(+, \mathrm{x})$ of Figs. 1a, 2a. Best fit is obtained for a hemispherical tip radius of $380 \AA$.

Figure 5. Schematic of the proposed kinetic model for field-induced mound formation. Radial electric field gradients result in preferential diffusion of adatoms toward the tip, resulting in diffusive fluxes, $F_{\text {in }}$ and $F_{\text {out }}$, into and out of an area element at radius, r. Field-enhanced detachment of adatoms at steps provides a source of adatoms, while reattachment of adatoms to steps provides a sink.

Figure 6. Calculated electric field and growth rate as a function of radius from the central axis for tip radius of $380 \AA$, tip bias of $-100 \mathrm{~V}$, and tip-sample separation of $250 \AA$.

Figure 7. Maximum electric field at the top of a mound growing under a tip. Tip radius is 380 $\AA$, tip bias is $-100 \mathrm{~V}$, and the gap between tip and planar substrate is $500 \AA$. 
Figure 8. Calculated maximum growth rate on a flat surface as a function of maximum electric field. 


\section{$\underline{\text { References }}$}

1 H. J. Mamin, P. H. Guethner, and D. Rugar, Physical Review Letters 65, 2418-2421 (1990).

2 H. J. Mamin, S. Chiang, H. Birk, P. H. Guethner, and D. Rugar, Journal of Vacuum Science and Technology B B9, 1398-1402 (1991).

3 H. J. Mamin and D. Rugar, in Scanning Probe Microscopy; Vol. 241, edited by H. K. Wickramasinghe (American Institute of Physics, New York, 1992), p. 462.

4 J. I. Pascual, J. Mendez, J. Gomez-Herrero, A. M. Baro, and N. Garcia, Physical Review Letters 71, 1852-1855 (1993).

5 H. J. Mamin and D. Rugar, Physical Review Letters 72, 1128-1128 (1994).

6 J. I. Pascual, J. Mendez, J. Gomez-Herrero, A. M. Baro, N. Garcia, and V. T. Binh, Physical Review Letters 72, 1129 (1994).

7 C. X. Guo and D. J. Thomson, Ultramicroscopy 42-44, 1452-1458 (1992).

8 T. C. Chang, C. S. Chang, H. N. Lin, and T. T. Tsong, Applied Physics Letters 67, 903-905 (1995).

9 L. Libioulle, Y. Houbion, and J.-M. Gilles, Journal of Vacuum Science and Technology B13, 1325-1331 (1995).

10 R. Koning, O. Jusko, L. Koenders, and A. Schlachetzki, Journal of Vacuum Science and Technology B 14, 48-53 (1996).

11 T. T. Tsong, Physical Review B 44, 13703-13710 (1991).

12 J. Mendez, J. Gomez-Herrero, J. I. Pascual, J. J. Saenz, J. M. Soler, and A. M. Baro, Journal of Vacuum Science and Technology B 14, 1145-1148 (1996).

13 T. T. Tsong and G. L. Kellogg, Physical Review B 12, 1343 (1975).

14 G. L. Kellogg and T. T. Tsong, Surf. Sci. 62, 343 (1977).

15 W. P. Dyke and W. W. Dolan, in Adv. in Electronics and Electron Physics; Vol. 8, edited by L. Marton (Academic Press, New York, 1956), p. 89.

16 J. Lyubinetsky, S. Mezhenny, and J. T. Yates, J. Vac. Sci. Technol. A in press (1999).

17 S. A. Joyce and J. E. Houston, Review of Scientific Instruments 62, 710-715 (1991).

18 S. A. Joyce, R. C. Thomas, J. E. Houston, T. A. Michalske, and R. M. Crooks, Physical Review Letters 69, 2790-2793 (1992).

19 P. Tangyunyong, R. C. Thomas, J. E. Houston, T. A. Michalske, R. M. Crooks, and A. J. Howard, Physical Review Letters 71, 3319-3322 (1993). 
20 P. Tangyunyong, R. C. Thomas, J. E. Houston, T. A. Michalske, R. M. Crooks, and A. J. Howard, Journal of Adhesion Science and Technology 8, 897-912 (1994).

21 R. C. Thomas, J. E. Houston, T. A. Michalske, and R. M. Crooks, Science 259, 18831885 (1993).

22 R. C. Thomas, P. Tangyunyong, J. E. Houston, T. A. Michalske, and R. M. Crooks, Journal of Physical Chemistry 98, 4493-4494 (1994).

23 R. C. Thomas, J. E. Houston, R. M. Crooks, T. Kim, and T. A. Michalske, Journal of the American Chemical Society 117, 3830-3834 (1995).

24 J. E. Houston, T. A. Michalske, and R. M. Crooks, in Interfacial Adhesion at the Molecular Level, Hilton-Head, SC, 1997 (The Adhesion Society), p. 31-33.

25 G. I. Taylor, Proceedings of the Royal Society London A280, 383-397 (1914).

26 R. Gomer, Applied Physics A 19, 365-375 (1979).

27 M. Lax, J. Appl. Phys. 48, 3919 (1977).

28 T. M. Mayer, D. P. Adams, and B. M. Marder, J. Vac. Sci. Technol. B 14, 2438 (1996).

29 CRC Handbook of Chemistry and Physics; Vol. , edited by D. R. Lide (CRC Press, Boca Raton, 1998).

30 K. L. Johnson, Contact Mechanics (Cambridge University Press, Cambridge, 1985).

31 J. D. Kiely and J. E. Houston, Phys. Rev. B 57, 57 (1998).

32 T. A. Michalske and J. E. Houston, Acta Materialia (1997).

33 G. L. Kellogg, Surface Science Reports 21, 1-88 (1994).

34 L. J. Whitman, J. A. Stroscio, R. H. Dragoset, and R. J. Celotta, Science 251, 1206 (1991).

35 C. Girard, C. Joachim, C. Chavy, and P. Sautet, Surf. Sci. 282, 400 (1993).

36 G. L. Kellogg, Phys. Rev. Lett. 70, 1631 (1993).

37 T. Kawai and K. Watanabe, Surf. Sci. 357-358, 830 (1996).

38 H. Cabibil, J. E. Houston, T. M. Mayer, and G. F. Franklin, Mater. Res. Soc. Proc. 522, 463 (1998).

39 G. L. Kellogg, private communication (1998).

40 B. S. Swartzentruber, A. P. Smith, and H. Jonsson, Phys. Rev. Lett. 77, 2518 (1996). 


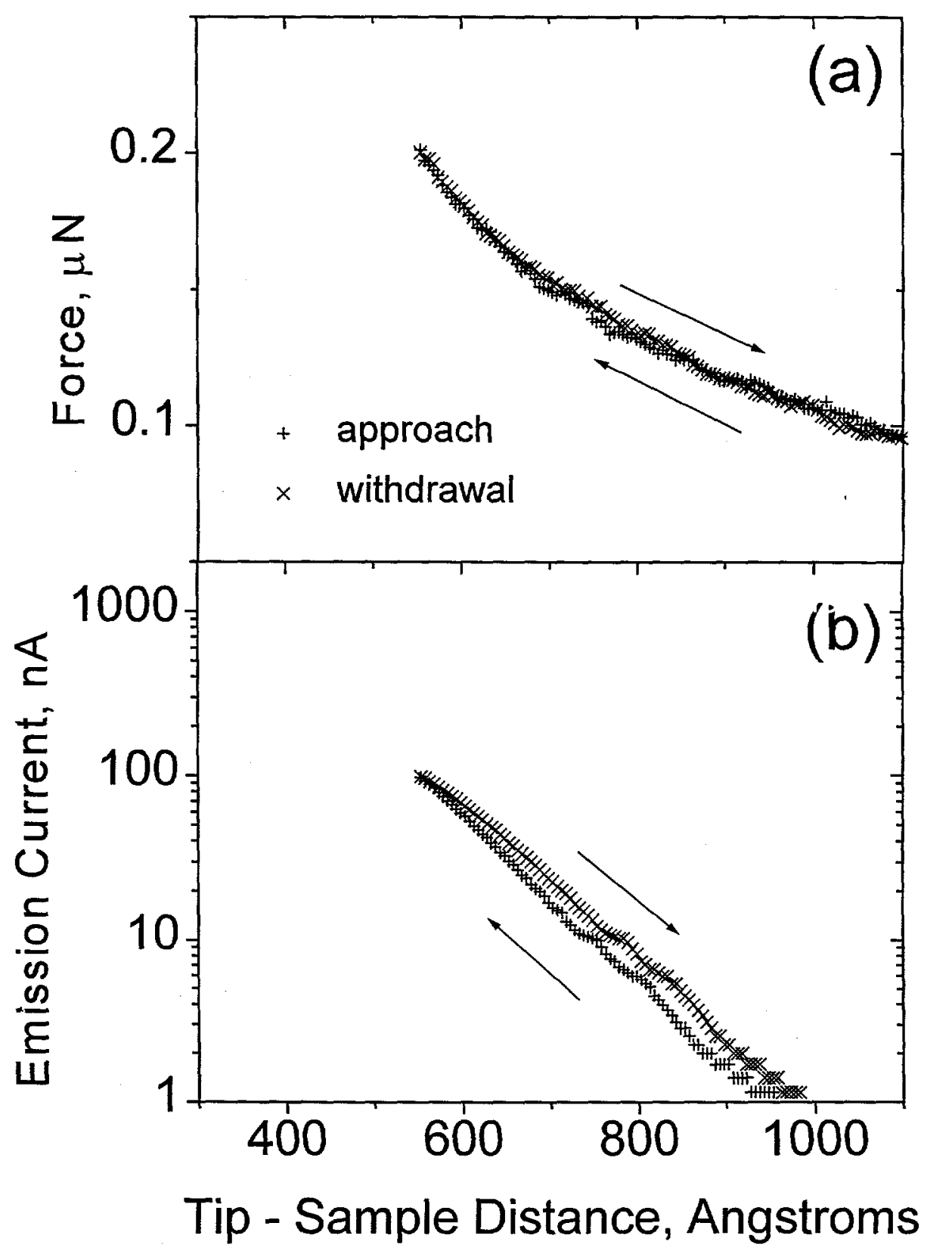

Fisl 


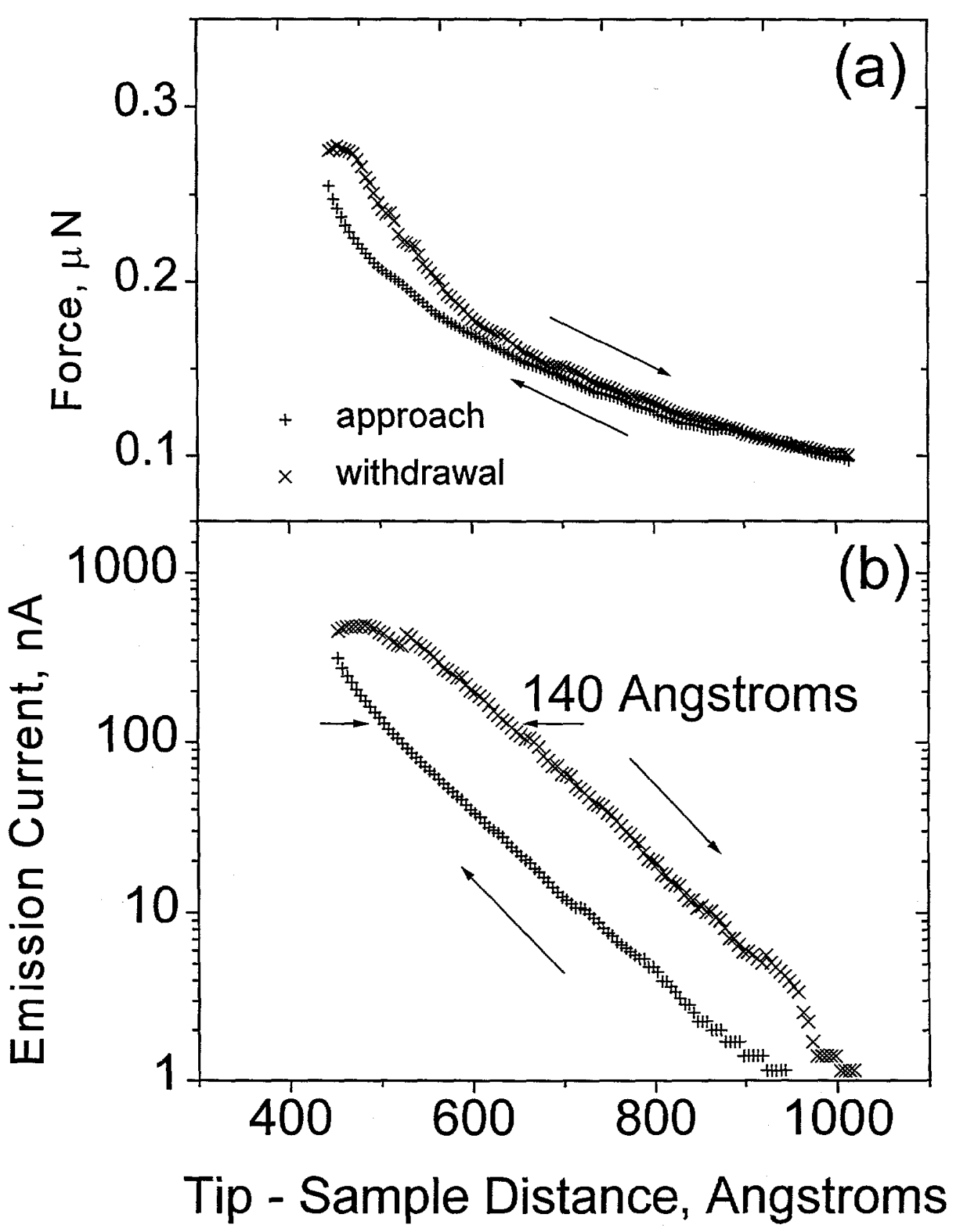

$F \cdot j 2$ 


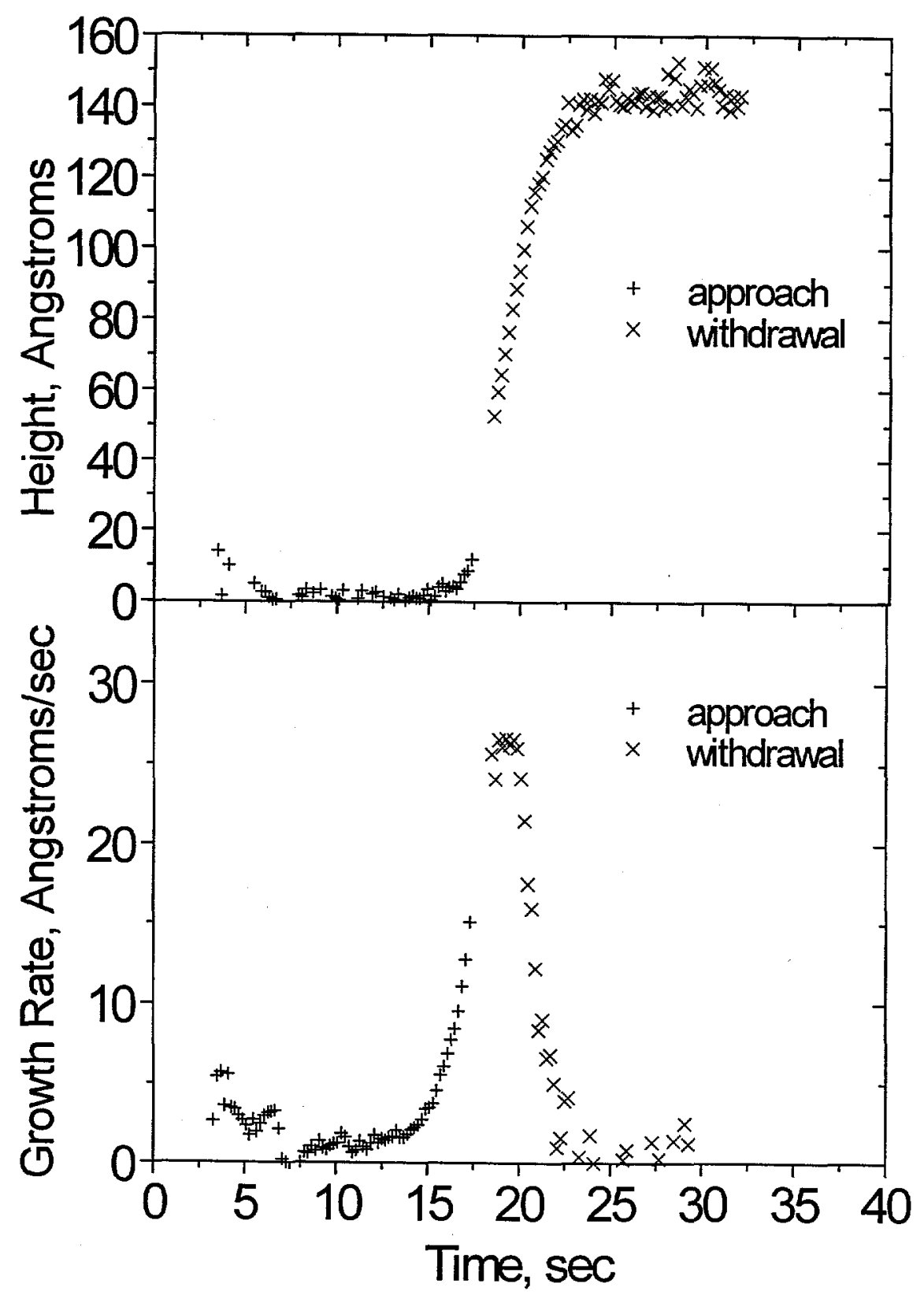

$F_{\text {is } 3}$ 


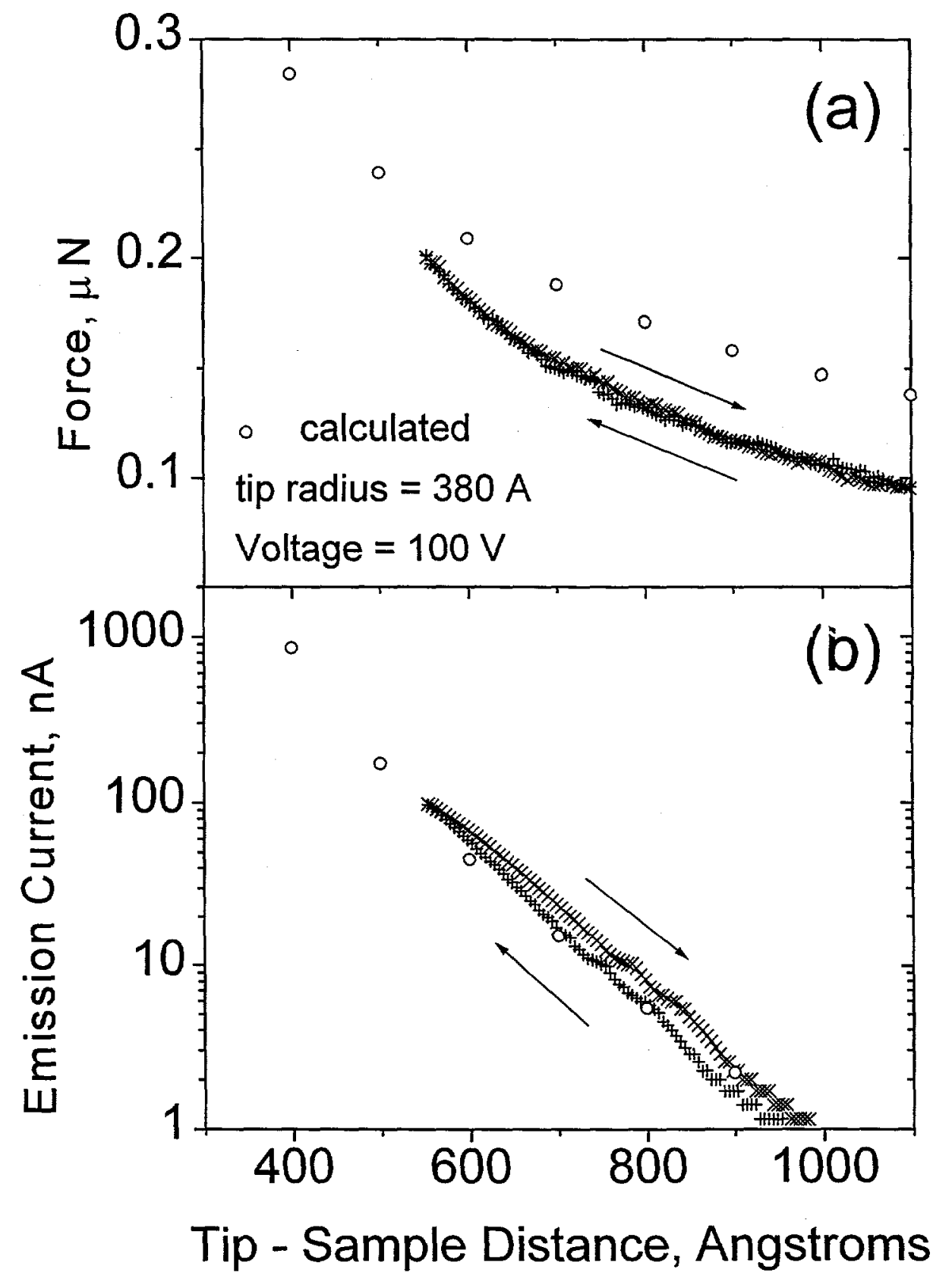

Fis 


\section{Diffusion}

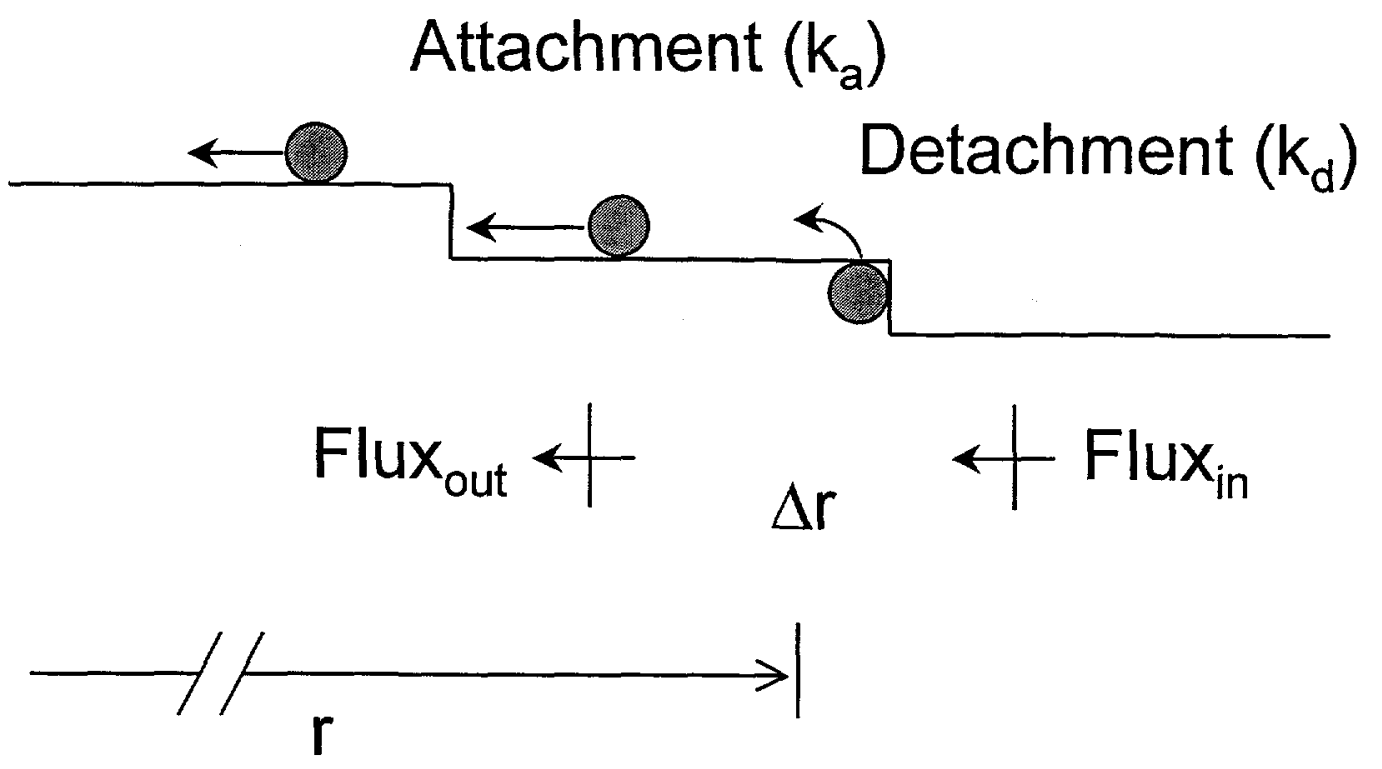

Fis 5 


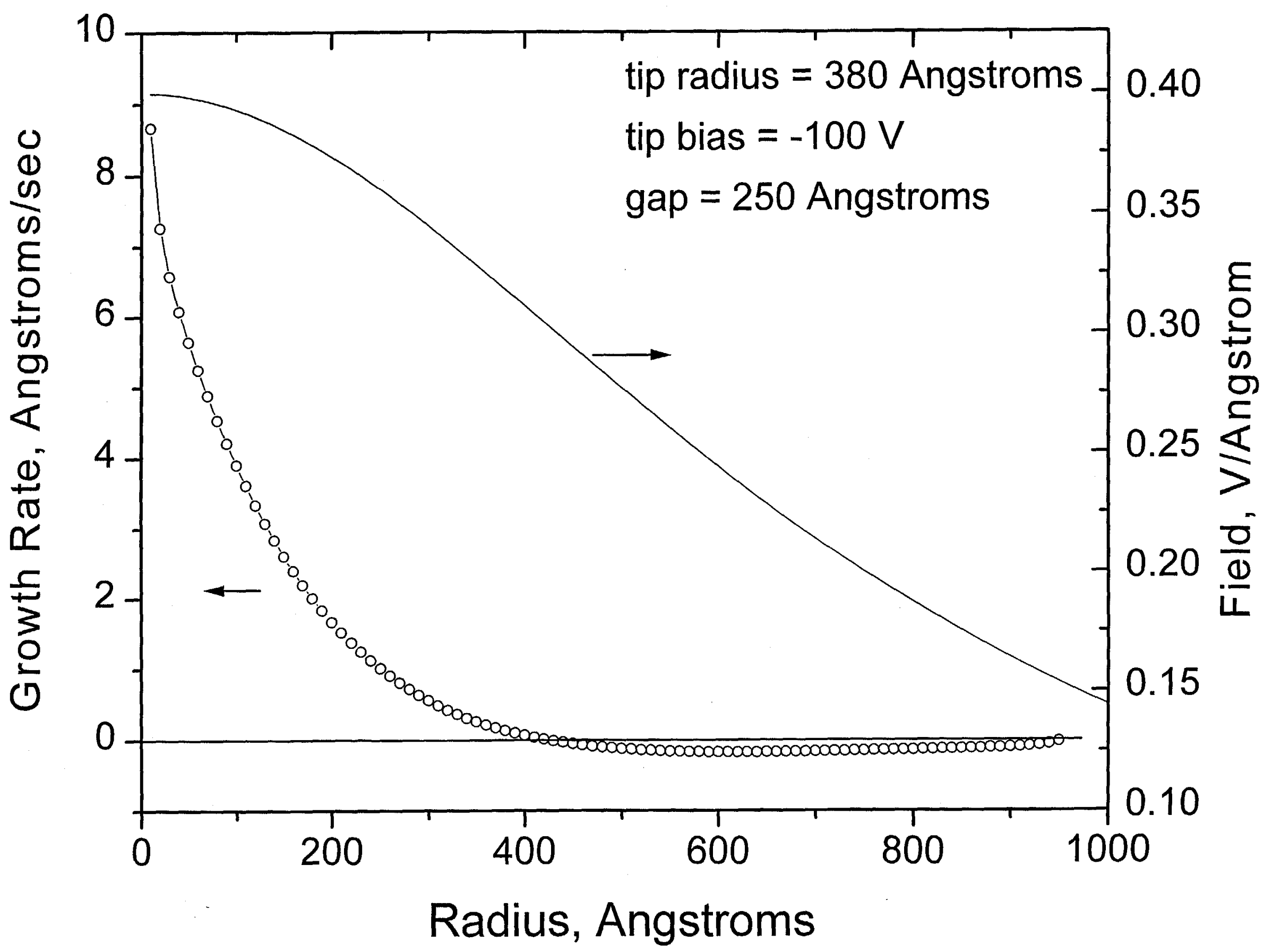




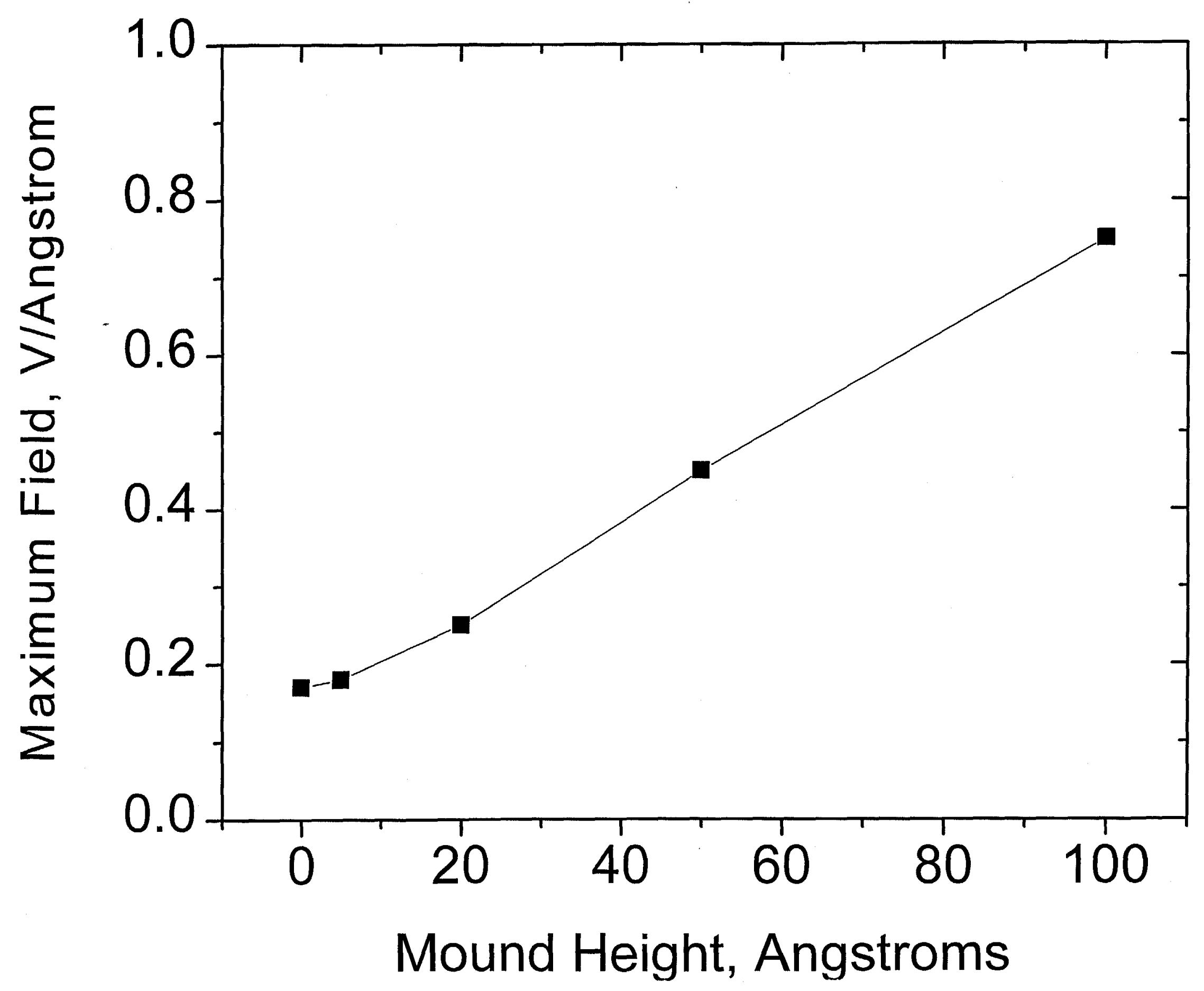




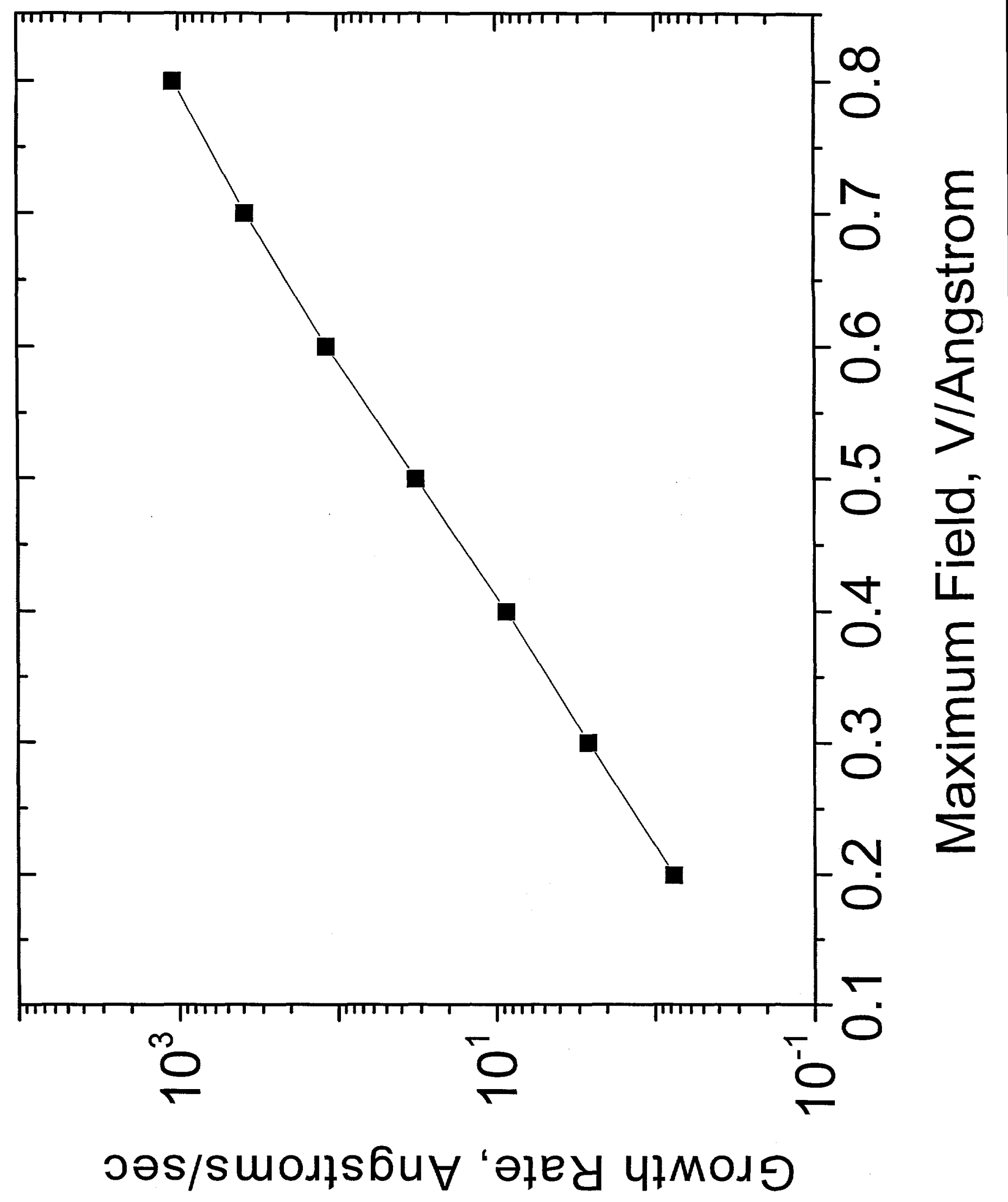

$F_{15}-8$ 